\title{
A simple vapor-diffusion method enables protein crystallization inside the HARE serial crystallography chip \\ Brenna Norton-Baker ${ }^{1}$, Pedram Mehrabi ${ }^{2}$, David von Stetten ${ }^{3}$, Hendrik Schikora ${ }^{4}$, Ashley O. Kwok ${ }^{5}$, Rachel W. Martin ${ }^{6}$, R. J. Dwayne Miller ${ }^{7}$, Eike C. Schulz ${ }^{8}$ \\ ${ }^{1}$ No affiliation given, ${ }^{2}$ Max Planck Institute for the Structure and Dynamics of Matter, ${ }^{3}$ European Molecular Biology Laboratory, ${ }^{4}$ Max-Planck-Institute for Structure and Dynamics of Matter, ${ }^{5}$ University of California, Irvine, ${ }^{6}$ University of California, Irvine, ${ }^{7}$ University of Toronto, ${ }^{8} \mathrm{Max}$ Planck Institute for the Structure and Dynamics of Matter bnortonb@uci.edu
}

Fixed-target serial synchrotron crystallography allows for the collection of thousands of diffraction snapshots on micrometer-sized crystals. Combined with reaction initiation techniques such as optical excitation or in situ mixing, serial crystallography enables time-resolved measurements and a deeper understanding of macromolecular dynamics during a reaction cycle. However, challenges remain for microcrystal preparation; previous studies have typically relied on batch crystallization, often requiring significant optimization and large sample volumes. Additionally, crystals must be transferred to the fixed target, resulting in physical stress that can be destructive to sensitive systems. In this work, we report a simple vapor-diffusion crystallization method that supports the growth of microcrystals inside the HARE serial crystallography chip. This in situ approach grows microcrystals inside the individual wells of the HARE chip equilibrated against a reservoir of mother liquor, easily translating crystallization conditions from the canonical hanging-drop method. Using this technique, microcrystals were grown in-chip with sufficient density to determine the structure for four different proteins: lysozyme, proteinase $\mathrm{K}$, xylose isomerase, and a new variant of the human eye lens proteins, $\gamma$ S-crystallin. The direct growth of microcrystals inside the chip eliminates physical handling of crystals, significantly reduces sample consumption, and simplifies the transition of crystallization conditions from hanging/sitting drop screening to microcrystallization for serial crystallography. 